\title{
Misalignment effects of segmented undulator in self-amplified spontaneous emission
}

\author{
T. Tanaka* and H. Kitamura \\ The Institute of Physical and Chemical Research, Koto 1-1-1, Mikazuki, Sayo, Hyogo 679-5148, Japan \\ T. Shintake \\ High Energy Accelerator Research Organization, 1-1 Oho, Tsukuba, Ibaraki, Japan
}

(Received 5 March 2002; published 8 April 2002)

\begin{abstract}
Effects caused by misalignment of undulator segments on the gain of a free electron laser (FEL) based on the process of self-amplified spontaneous emission are theoretically studied. The well-known FEL equations are solved analytically and numerically with boundary conditions describing the misalignment effect in order to obtain the amplification gain as a function of the distance from the undulator entrance. The two worst cases of alignment are considered. One is that the phase slip over the drift section between the adjacent segments is $\pi$, and the other is that the radiation is cut off at the drift section. Calculations show that if the undulator segment is several times as long as the gain length, the radiation power is close to that of no segmentation in both cases.
\end{abstract}

DOI: 10.1103/PhysRevSTAB.5.040701

\section{INTRODUCTION}

In $\mathrm{x}$-ray free electron laser (FEL) projects based on the process of self-amplified spontaneous emission (SASE), e.g., Linac Coherent Light Source [1], TESLA [2], and SPring-8 Compact SASE Source [3], a long undulator (typically longer than $20 \mathrm{~m}$ ) is to be installed. In such a case, the undulator is usually divided into several segments to provide drift sections for installation of focusing magnets and beam-diagnostics instruments. In order to realize the x-ray FEL, the whole undulator should have good performances as well as the accelerator.

To date, a great number of undulators have been constructed and installed in many synchrotron radiation facilities, which has improved the technologies necessary for undulator development. The support structure has good mechanical tolerances and the magnetic fields are precisely measured and finely corrected. By means of these technologies, it is possible to construct one undulator segment close to an ideal device with low phase errors and electronorbit deviations.

What is more important to realize the $\mathrm{x}$-ray FEL is an alignment of each segment. The electron path length over the drift section should be adjusted (longitudinal alignment) to obtain good phase matching between radiation fields emitted from adjacent segments. The electron deflection and displacement should be kept small (transverse alignment) to ensure overlap of the electron and photon beam.

For the longitudinal alignment, optimization of the end structure of the undulator magnet is necessary. Furthermore, the undulator gap should be fixed to ensure the phase-matching condition, because the phase slip over the

*Electronic address: ztanaka@spring8.or.jp
PACS numbers: 41.60.Cr

drift section is a function of the magnetic strength of the undulator. For the transverse alignment, steering magnets placed in the drift section or alternative schemes are used to correct the electron orbit with the beam-based alignment technique $[4,5]$ that uses beam position monitor readings as a function of deliberate variations in the electron energy. Thus, both alignments cause a lot of trouble in the operation of the accelerator.

Here we pose one question. Is the alignment really necessary? What is important for the amplification process in SASE is the density modulation or bunching in the electron beam. Even if the phase matching or overlap between the photon and electron beams is completely lost at the drift section due to bad alignment, the density modulation is conserved. We can therefore expect much more intense radiation than is obtained for the spontaneous emission. Under several conditions, the intensity may be close to that obtained for the ideal FEL. In order to discuss the problem concretely, let us consider simply the amplification process of FEL. The electric field of radiation in FEL is roughly scaled as $e^{\sqrt{3} z / 2 L g}$ [6] where $z$ is the distance from the undulator entrance and $L_{g}$ the gain length. If the length of one segment is 5 times as long as $L_{g}$, the power at the exit of the segment is calculated as $e^{5 \sqrt{3}}=5770$ times as large as that at the entrance. This means that the radiation emitted from the preceding segment contributes little to the total power. In other words, we can expect the same radiation intensity regardless of the optical relation between segments.

The qualitative discussions above suggest that if one segment of the undulator is sufficiently long compared to the gain length, neither longitudinal nor transverse alignment is important; rather, the radiation from the upstream can be cut off to stabilize the operation. The purpose of this article is to investigate quantitatively the effect of misalignment. The two worst cases are considered. One is that the overlap of the electron and photon beams is completely lost 
due to the transverse misalignment, and the other is that the phase slip over the drift sections between adjacent segments is equal to $\pi$ due to the longitudinal misalignment. We call the former optical cutoff because the photon beam seems to be intercepted by an optical shutter at the drift sections and the latter phase flip.

\section{THEORY}

Let us consider an undulator composed of several segments with the length of $L$ and drift sections in between as shown in Fig. 1. The phase-slip error (longitudinal misalignment) and degradation of the electric field (transverse misalignment) are assumed to be the only effects caused by the drift sections on the amplification process. Other possible effects are neglected because they are small as shown by Kim et al. [7,8]. All equations are written in the one-dimensional case using SI units. Also, the initial energy spread of the electron beam is neglected to simplify the problem. Three-dimensional and energy-spread effects will result in the lengthening of the gain length $L_{g}$ to be introduced later. The three-dimensional gain length $L_{g, 3 \mathrm{D}}$ with the energy spread taken into account can be evaluated by the universal scaling function developed by Xie et al. [9] and is about 1.1-2 times longer than $L_{g}$. Thus, the following discussions can be applied to the case when the three-dimensional and energy-spread effects are taken into account, by replacing $L_{g}$ with $L_{g, 3 \mathrm{D}}$.

\section{A. FEL equations}

The equations describing the FEL process are [10]

$$
\begin{gathered}
\gamma_{j}^{\prime}=-\frac{2 k_{u} D_{1}}{\gamma_{0}}\left(E e^{i \psi_{j}}+\text { c.c. }\right), \\
\psi_{j}^{\prime}=\frac{2 k_{u}\left(\gamma_{j}-\gamma_{0}\right)}{\gamma_{0}}-\Delta k_{u}, \\
E^{\prime}=\frac{k_{u} D_{2}}{2 \gamma_{0}}\left\langle e^{-i \psi_{j}}\right\rangle
\end{gathered}
$$

with

$$
\begin{gathered}
D_{1}=\frac{e K[J J]}{2 m c^{2} k_{u}}, \\
D_{2}=\frac{n_{0} \mu_{0} e c^{2} K[J J]}{k_{u}}, \\
\longrightarrow \text { Undulator }
\end{gathered}
$$

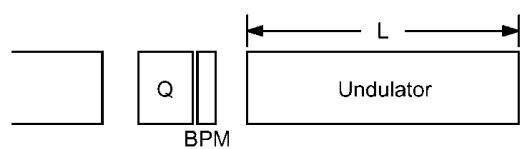

FIG. 1. Undulator composed of several segments with the length of $L$. In the drift sections, focusing magnets (indicated by Q) and beam position monitors (BPM) are installed.

$$
\begin{gathered}
{[J J]=J_{0}\left(\frac{K^{2} / 4}{1+K^{2} / 2}\right)-J_{1}\left(\frac{K^{2} / 4}{1+K^{2} / 2}\right),} \\
\Delta k_{u}=k_{u}\left(\frac{\omega}{\omega_{1}}-1\right), \\
\omega_{1}=\frac{4 \pi c \gamma^{2}}{\lambda_{u}\left(1+K^{2} / 2\right)},
\end{gathered}
$$

where $E$ is the electric field of the amplified radiation, $\gamma_{j}$ and $\psi_{j}$ the energy and phase of the $j$ th electron, $K$ and $2 \pi / k_{u}$ the deflection parameter and periodic length of the undulator, $n_{0}$ the number of electrons per unit volume, $\omega$ the energy of the amplified radiation, and the prime denotes differentiation with respect to $z$, the distance along the undulator axis.

Now let us consider the case with a certain phase jump $\Delta \psi$ existing at the drift space. In this case, the phase growth equation (2) should be modified to

with

$$
\Delta(z)=\sum_{n=1}^{\infty} \delta(z-n L) \Delta \psi,
$$

where $\delta(z)$ is a delta function. Let us introduce a new phase

$$
\phi_{j}=\psi_{j}-\Theta(z)+\Delta k_{u},
$$

with

$$
\Theta(z)=\sum_{n=1}^{\infty} \theta(z-n L) \Delta \psi,
$$

where $\theta(z)$ is a step function. Then the FEL equations (1)-(3) can be modified as follows:

$$
\gamma_{j}^{\prime}=-\frac{2 k_{u} D_{1}}{\gamma_{0}}\left(E e^{i \Theta(z)-i \Delta k_{u} z} e^{i \phi_{j}}+\text { c.c. }\right),
$$

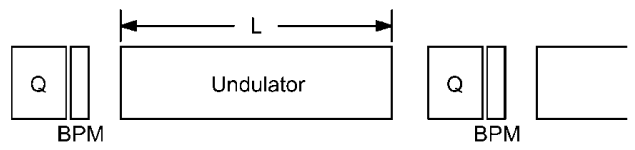

$$
\psi_{j}^{\prime}=\frac{2 k_{u}\left(\gamma_{j}-\gamma_{0}\right)}{\gamma_{0}}-\Delta k_{u}+\Delta(z),
$$




\section{B. Analytical solution}

Now let us solve analytically the modified FEL equations (4)-(6) within the framework of linear approximation.

Multiplying Eq. (6) by $e^{i \Theta(z)}$ and differentiating twice, we have the third-order ordinary differential equation

$$
\begin{aligned}
\left(E^{\prime} e^{i \Theta(z)}\right)^{\prime \prime}-2 i \Delta k_{u}\left(E^{\prime} e^{i \Theta(z)}\right)^{\prime}- & \\
\Delta k_{u}^{2}\left(E^{\prime} e^{i \Theta(z)}\right) & =i\left(2 \rho k_{u}\right)^{3}\left(E e^{i \Theta(z)}\right),
\end{aligned}
$$

with

$$
(2 \rho)^{3}=\frac{2 D_{1} D_{2}}{\gamma_{0}^{3}},
$$

where we have neglected the term $\left(\phi_{j}\right)^{2}$ as being second order and changed the variable from $z$ to $\tau$ defined by

$$
\tau=\frac{z}{L_{g}}
$$

with

$$
L_{g}=\frac{1}{2 \rho k_{u}}
$$

being the gain length in one-dimensional form. The quantity $\rho$ is known as a FEL parameter and usually of the order of $10^{-3}$.

Let us look for the general solution within one segment. In this case $\Theta(z)$ is constant, therefore Eq. (7) is simplified to

$$
E^{\prime \prime \prime}-2 i\left(\frac{\Delta k_{u}}{2 \rho k_{u}}\right) E^{\prime \prime}-\left(\frac{\Delta k_{u}}{2 \rho k_{u}}\right)^{2} E^{\prime}=i E .
$$

The general solution of Eq. (8) is given by a superposition of three linearly independent solutions [11]

$$
E=\sum_{j=1}^{3} C_{j} e^{\lambda_{j} \tau}
$$

with $\lambda_{j}$ being a solution of the dispersion relation

$$
\lambda\left(\lambda+i \frac{\Delta k_{u}}{2 \rho k_{u}}\right)^{2}=i .
$$

The coefficients $C_{j}$ are determined by the initial conditions

$$
\left(\begin{array}{c}
E_{0} \\
E_{0}^{\prime} \\
E_{0}^{\prime \prime}
\end{array}\right)=\left(\begin{array}{ccc}
1 & 1 & 1 \\
\lambda_{1} & \lambda_{2} & \lambda_{3} \\
\lambda_{1}^{2} & \lambda_{2}^{2} & \lambda_{3}^{2}
\end{array}\right)\left(\begin{array}{l}
C_{1} \\
C_{2} \\
C_{3}
\end{array}\right)
$$

The solution in the $n$th segment is therefore expressed as

$$
\left(\begin{array}{c}
E \\
E^{\prime} \\
E^{\prime \prime}
\end{array}\right)=M(z)\left(\begin{array}{c}
E_{n 0} \\
E_{n 0}^{\prime} \\
E_{n 0}^{\prime \prime}
\end{array}\right)
$$

where $E_{n 0}$ is the electric field at the entrance of the $n$th segment and the matrix $M(z)$ is defined as

$M(z)=\left(\begin{array}{ccc}e^{\lambda_{1} z} & e^{\lambda_{2} z} & e^{\lambda_{3} z} \\ \lambda_{1} e^{\lambda_{1} z} & \lambda_{2} e^{\lambda_{2} z} & \lambda_{3} e^{\lambda_{3} z} \\ \lambda_{1}^{2} e^{\lambda_{1} z} & \lambda_{2}^{2} e^{\lambda_{2} z} & \lambda_{3}^{2} e^{\lambda_{3} z}\end{array}\right)\left(\begin{array}{ccc}1 & 1 & 1 \\ \lambda_{1} & \lambda_{2} & \lambda_{3} \\ \lambda_{1}^{2} & \lambda_{2}^{2} & \lambda_{3}^{2}\end{array}\right)^{-1}$.

Now let us consider the boundary condition between segments. To satisfy Eq. (6), the derivatives of $E$ should have discontinuous boundary conditions

$$
\begin{aligned}
& \lim _{z \rightarrow n L+0} E^{\prime}(z)=e^{i \Delta \psi} \lim _{z \rightarrow n L-0} E^{\prime}(z), \\
& \lim _{z \rightarrow n L+0} E^{\prime \prime}(z)=e^{i \Delta \psi} \lim _{z \rightarrow n L-0} E^{\prime \prime}(z),
\end{aligned}
$$

while $E$ has

$$
\lim _{z \rightarrow n L+0} E(z)=\eta \lim _{z \rightarrow n L-0} E(z),
$$

where $\eta$ denotes the cutoff effect due to transverse misalignment. Finally, we have the solution for the whole undulator

$$
\left(\begin{array}{c}
E \\
E^{\prime} \\
E^{\prime \prime}
\end{array}\right)_{z}=\mu\left(\begin{array}{c}
E_{0} \\
E_{0}^{\prime} \\
E_{0}^{\prime \prime}
\end{array}\right)
$$

with

$$
\mu=M(z-n L)\left[\left(\begin{array}{ccc}
\eta & 0 & 0 \\
0 & e^{i \Delta \psi} & 0 \\
0 & 0 & e^{i \Delta \psi}
\end{array}\right) M(L)\right]^{n} .
$$

Let us consider the initial condition where there is no electromagnetic field but the electron beam is modulated in density at the entrance. In this case we have

$$
\begin{gathered}
E_{0}=0, \\
E_{0}^{\prime}=\frac{k_{u} D_{2}}{2 \gamma_{0}}\left\langle e^{i \phi_{j}}\right\rangle_{0}, \\
E_{0}^{\prime \prime}=\frac{k_{u} D_{2}}{2 \gamma_{0}} i \Delta k_{u}\left\langle e^{-i \phi_{j}}\right\rangle_{0} .
\end{gathered}
$$

The last two formulas are derived from Eq. (6) and its derivative. Substituting into Eq. (12), the electric field is calculated as

$$
E=\left(\frac{D_{2}}{4 \rho \gamma_{0}}\right)\left(\mu_{12}+i \frac{\Delta k_{u}}{2 \rho k_{u}} \mu_{13}\right)\left\langle e^{-i \phi_{j}}\right\rangle,
$$

where $\mu_{12,13}$ are the elements of the matrix $\mu$. In the case of SASE, the modulation term $\left\langle e^{i \phi_{j}}\right\rangle$ plays a role of the input signal and the electric field is expressed as a superposition of the plane wave $E$. In other words, it is given by the Fourier transform

$$
E(z, t)=\frac{1}{2 \pi} \int_{-\infty}^{\infty} E_{\omega}(z) e^{-i \omega t} d \omega
$$


with

$$
\begin{gathered}
E_{\omega}=S(\omega, z) \frac{I_{\omega}}{I_{0}} E_{0}, \\
S(\omega, z)=\mu_{12}+i \frac{\Delta k_{u}}{2 \rho k_{u}} \mu_{13}, \\
E_{0}=\frac{D_{2}}{4 \rho \gamma_{0}},
\end{gathered}
$$

where $I_{\omega}$ is the Fourier transform of the electron beam current and $I_{0}$ the average beam current. The power density is calculated as

$$
\frac{d P}{d S}=\frac{\epsilon_{0} c}{\pi T} \int_{0}^{\infty}\left\langle\left|E_{\omega}(z)\right|^{2}\right\rangle d \omega .
$$

Let us define a normalized power $\kappa$ as the power of the amplified radiation normalized by the electron beam power calculated as

$$
\kappa=\frac{\rho}{N_{\lambda}} \int_{0}^{\infty}|S(\omega, z)|^{2} \frac{d \omega}{\omega_{1}},
$$

with

$$
N_{\lambda}=\frac{2 \pi I_{0}}{e \omega_{1}}
$$

being the number of electrons per resonance wavelength, $\lambda_{1}=2 \pi c / \omega_{1}$.

\section{Numerical integration}

In order to investigate the amplification process near saturation, the FEL equations (1)-(3) are solved numerically with boundary conditions (9)-(11) and initial conditions described later being taken into account. It should be noted that the SASE process is time dependent, i.e., the amplified radiation advances the electron by the resonance wavelength while the electron travels the periodic length of the undulator, $\lambda_{u}=2 \pi / k_{u}$. We therefore divide the electron bunch into many boxes with the length of $\lambda_{1}$, solve the FEL equations in each box to obtain the amplified field, and then transfer it into the preceding box after advancing the electron beam by $\lambda_{u}$ [12]. The normalized power is obtained by averaging the power in all boxes, and the spectrum is obtained by Fourier transformation of the electric field in each box.

In SASE simulation, the initial condition of the density modulation, or the shot noise, should be taken into account carefully. Ideally, the FEL equations should be solved for all the electrons in the beam bunch. Needless to say, it is impractical to perform such a computation because of the limited CPU time. We have to substitute a limited number of macroparticles for all the electrons. In order to obtain a reasonable result with less CPU time, we have adopted the procedure developed by Penman et al. [13]. Because the electron has a random phase, the values $\left|\left\langle e^{-i \phi_{j}}\right\rangle\right|^{2}$ in each box will be distributed with the negative exponential distribution given by

$$
p\left(\left|\left\langle e^{-i \phi_{j}}\right\rangle\right|^{2}\right)=\frac{1}{N_{\lambda}} \exp \left(-\frac{\left|\left\langle e^{-i \phi_{j}}\right\rangle\right|^{2}}{N_{\lambda}}\right) .
$$

In order to reproduce this distribution, the initial electron phase is determined by the equation

$$
\phi_{j}=\frac{2 \pi}{N_{m}}\left(j-\frac{1}{2}\right)+(2 r-1) \sqrt{\frac{3 N_{m}}{N_{\lambda}}},
$$

where $r$ is a pseudorandom number uniformly distributed between 0 and 1 , and $N_{m}$ is a number of macroparticles in each box.

\section{NUMERICAL RESULTS}

Now let us investigate the misalignment effect of the segmented undulator in SASE using the analytical formulas derived in Sec. II B and the numerical solution described in Sec. IIC. The parameters considered are summarized in Table I. The total length of the undulator is assumed to be $15 L_{g}$ and four cases of numbers of segments are considered: $1,3,10$, and 15 . If the entire bunch is taken into account, the number of boxes to be considered, $N_{b}$, is calculated as $0.15(\mathrm{~mm}) / 3.6(\mathrm{~nm})=4.2 \times 10^{4}$. In order to save the computation time, we have reduced $N_{b}$ to 500 and confirmed that applying a longer bunch length does not change the result significantly.

\section{A. Optical cutoff}

First the optical cutoff effect is considered. In this case, both $\eta$ and $\Delta \psi$ are equal to 0 . Figure 2 shows the dependence of the normalized power $(\kappa / \rho)$ on the normalized distance from the undulator entrance $(\tau)$ for different numbers of undulator segments calculated with the two methods, i.e., analytical and numerical solutions of FEL equations.

In each figure, the numerical solutions in the exponential gain regime are in good agreement with the analytical ones. In the case of no segmentation, the normalized power saturates around $\tau=13$ and has the maximum value of $\rho$. The discontinuity observed at the drift section between segments is due to the optical cutoff. In the case

TABLE I. Electron beam and undulator parameters. The number of boxes $\left(N_{b}\right)$ and macroparticles $\left(N_{m}\right)$ used for numerical integration are also shown.

\begin{tabular}{lc}
\hline Electron energy & $1 \mathrm{GeV}$ \\
Bunch charge & $1 \mathrm{nC}$ \\
Bunch length & $0.15 \mathrm{~mm}$ \\
Energy spread & 0 \\
Period length & $15 \mathrm{~mm}$ \\
$K$ value & 1.3 \\
Total length & $20 \mathrm{~m}$ \\
$\lambda_{1}$ & $3.6 \mathrm{~nm}$ \\
$L_{g}$ & $1.33 \mathrm{~m}$ \\
$\rho$ & $8.9 \times 10^{-4}$ \\
$N_{b}$ & 500 \\
$N_{m}$ & 100 \\
\hline \hline
\end{tabular}




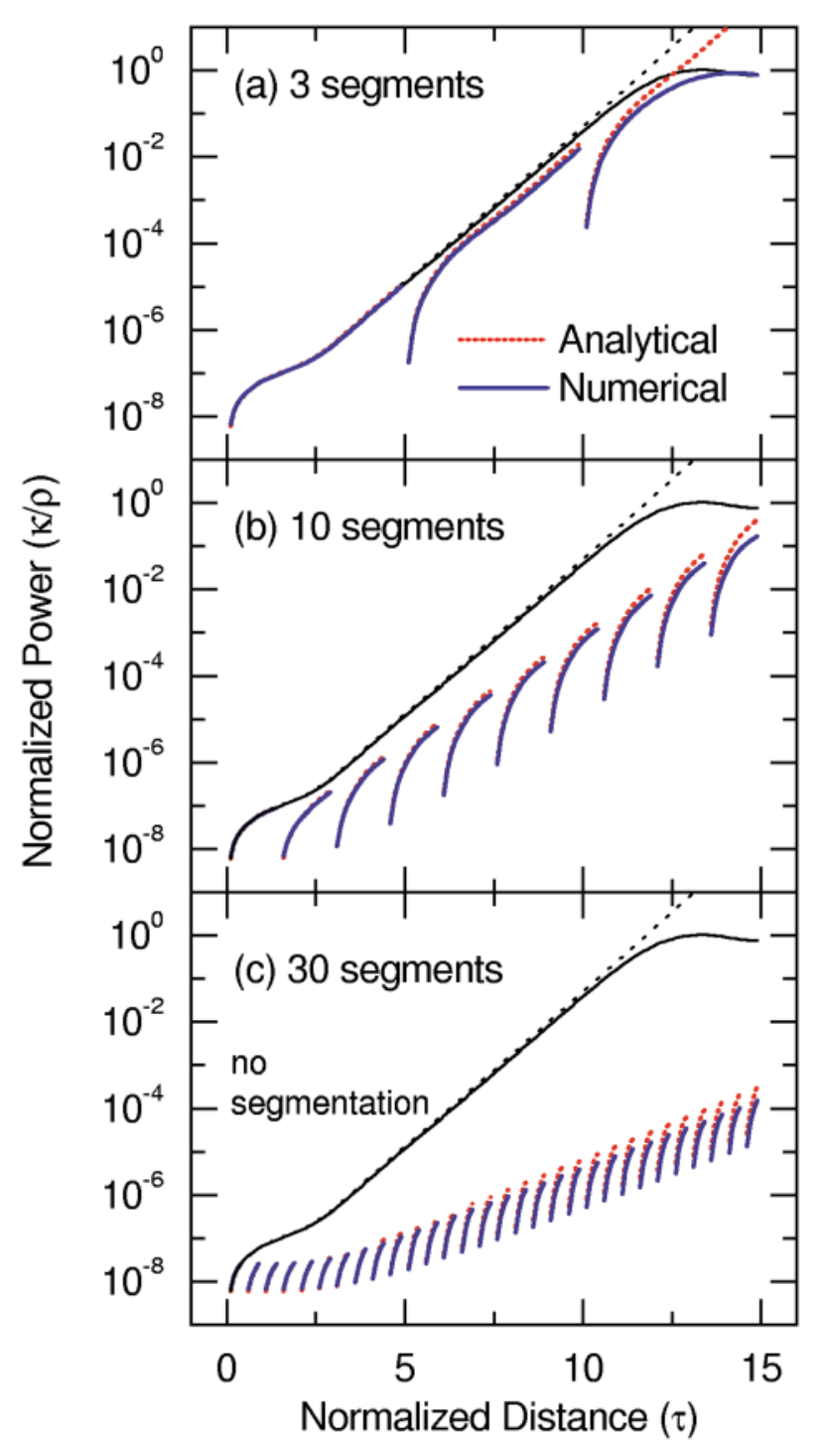

FIG. 2. (Color) Normalized power $\kappa / \rho$ versus the normalized distance $\tau$ in the optical cutoff case for different numbers of segments: (a) 3 segments, (b) 10 segments, and (c) 30 segments. The discontinuity corresponds to the drift section. In each figure, the no-segmentation case is also shown for comparison.

of 30 segments, the power at $\tau=13$ is lower than the nosegmentation case by an order of 4 . In the case of three segments, however, the radiation power is restored soon after the cutoff and the saturation length is about $\tau=14$, just a little longer than the no-segmentation case.

\section{B. Phase flip}

Next the phase-flip effect is considered. In this case, $\eta$ and $\Delta \psi$ are equal to unity and $\pi$, respectively. Figure 3 shows the dependence of $\kappa / \rho$ on $\tau$ for different numbers of undulator segments. Again, the numerical solutions are in good agreement with the analytical ones.

For the 3-segment case, we can observe a small dip soon after the drift space. Clearly the radiation power is

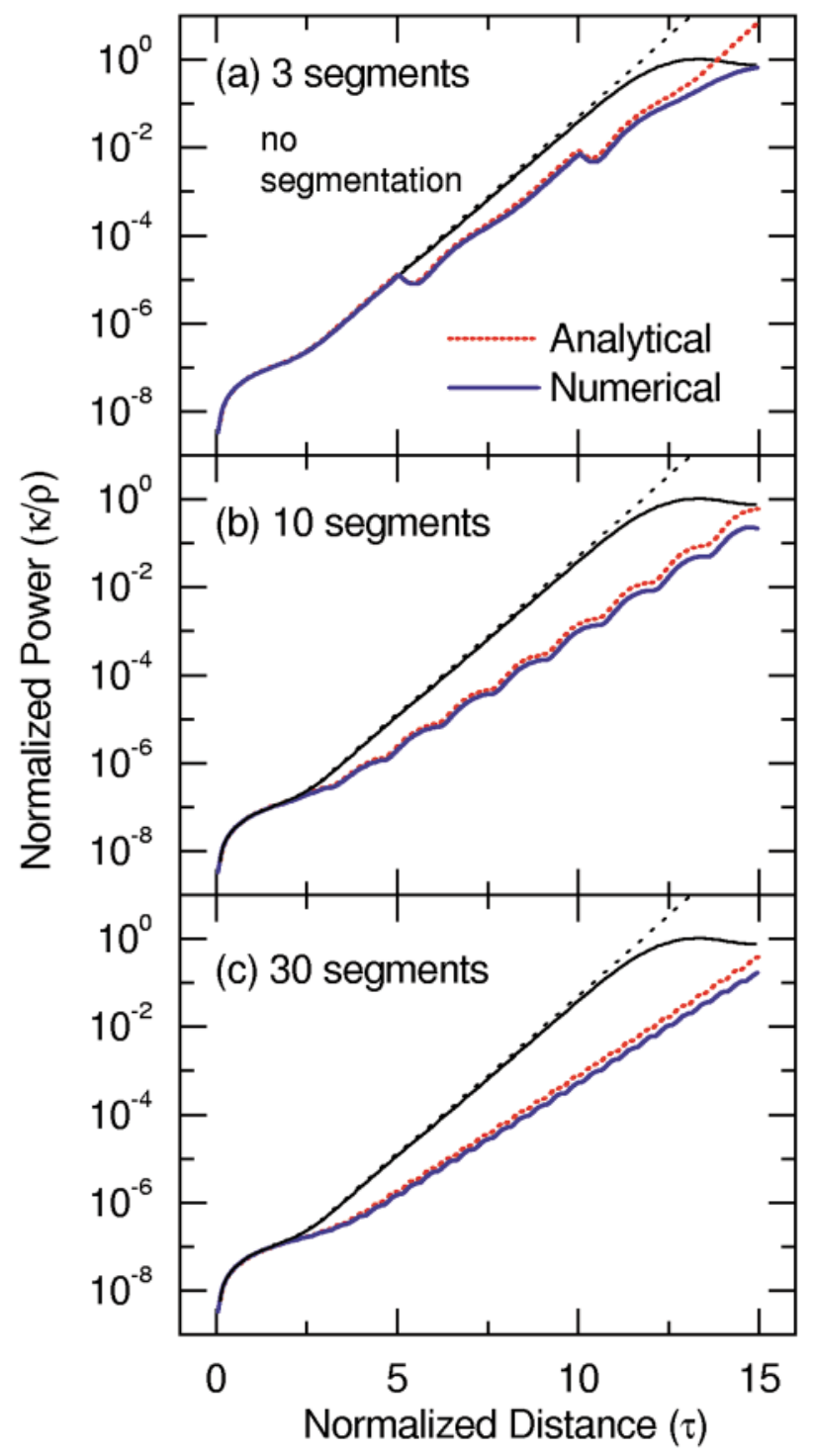

FIG. 3. (Color) Same as Fig. 2, but for the phase-flip case.

dissipated to accelerate the electrons. In all cases of segmentation, the efficiency does not saturate before the end of the undulator; however it should be noted that amplification occurs in spite of the antiresonance condition. We can find the reason in Fig. 4 where the spectrum of the amplified radiation, or the dependence of $\left|E_{\omega}\right|^{2}$ on $\Delta k_{u} /\left(2 \rho k_{u}\right)$, is plotted for different values of $\tau$. The spectrum is normalized so that the power obtained by integration over $\Delta k_{u}$ is equal to unity. In the case of no segmentation, the radiation around the resonance energy $\left(\Delta k_{u}=0\right)$ is amplified. On the other hand, no amplification takes place at the resonance energy in the case of 30 segments because of the antiresonance condition, while there are two different energies where amplification occurs. However, such a peak split is in general undesirable for the user of the FEL. In any case, the number of segments should be as small as possible to obtain high gain. 


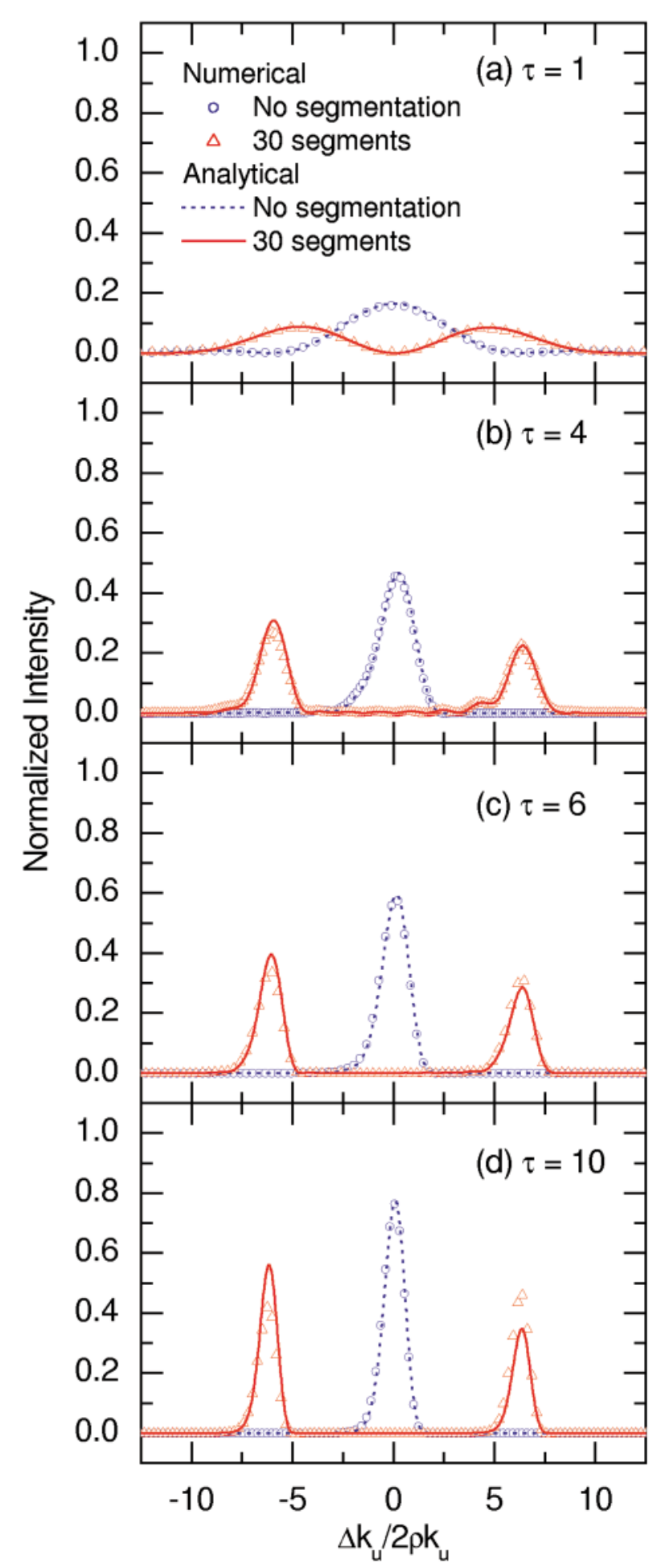

FIG. 4. (Color) Growth of radiation spectrum. The normalized spectrum is shown for different distances from the undulator entrance: (a) $\tau=1$, (b) $\tau=4$, (c) $\tau=6$, and (d) $\tau=10$. Two cases with no segmentation and 30 segments are shown.

\section{Achievable power}

In order to determine the minimum length per segment which can release us from the alignment troubles, let us

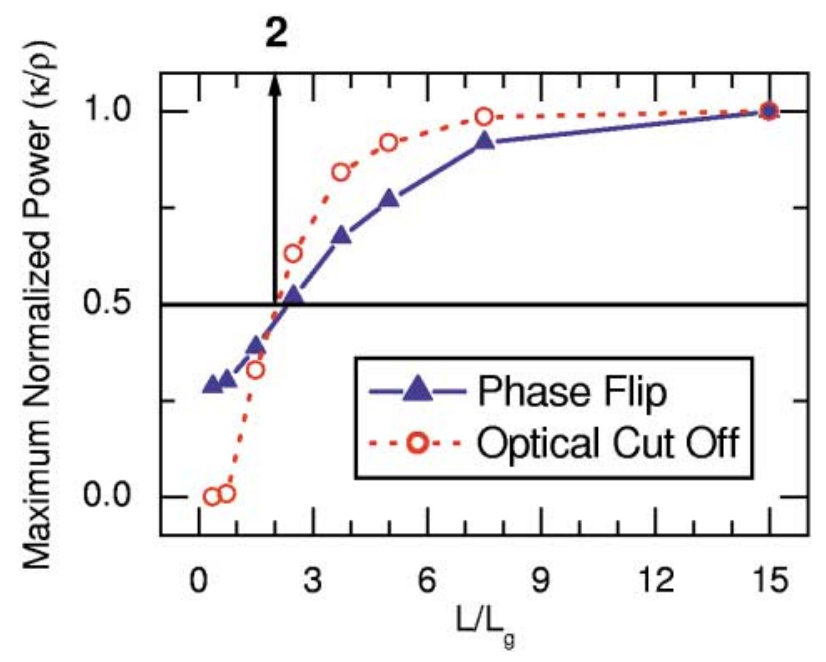

FIG. 5. (Color) Achievable power as a function of the undulator length per segment. The two cases of misalignment are shown.

calculate the maximum power obtained from the FEL system. Let us call it achievable power. We should consider two cases. If the FEL radiation is before saturation, the achievable power is equal to the power at the undulator exit, while it is regarded as the saturation power after saturation. Figure 5 shows the achievable power as a function of the undulator length per segment $(L)$. If $L$ is longer than $2 L_{g}$, achievable power higher than $0.5 \rho$ is obtained. This is one-half of the saturation power $\rho$. Therefore, we can regard $L>2 L_{g}$ as the condition which allows us to omit both alignments. Let us call hereafter the undulator segment long if this condition is satisfied, and short if not.

\section{DISCUSSION}

Now let us consider the practical case of misalignment. Possible sources of the transverse misalignment are error magnetic fields of the undulator and misalignment of the focusing magnets placed in the drift section. In fact, careful measurement and correction of the magnetic field will considerably reduce the field error of the undulator, while it may be difficult to align the focusing magnets within an accuracy required so that the system can work as the FEL. Thus, the possible transverse misalignment will exist at the drift sections, which, in the worst case, leads to the optical cutoff already discussed in Sec. III A. As for the longitudinal misalignment, the only possible source is the phase-slip variation due to the gap change, if the undulator segments are placed carefully.

If the length per segment is short, we have to correct both misalignments. This means that the focusing magnets should be aligned or the magnetic fields of the steering coils placed in the drift sections should be determined with the beam-based alignment to correct the transverse misalignment. The undulator gap should be fixed at the 
adequate gap where the phase slip is an integer multiple of $2 \pi$ to avoid the longitudinal misalignment.

On the other hand, we do not have to take care of both misalignments if the length per segment is long. This means that we do not have to perform the beam-based alignment and are free from restrictions on the available gap. The lasing wavelength therefore can be tuned by changing the gap instead of the electron energy. If, like SCSS, an in-vacuum undulator [14] is adopted, the inner vertical aperture is not limited by the vacuum vessel and is identical to the undulator gap, which means that the vertical aperture is also variable. Therefore, if the resistivewall effect is found to be a problem during operation, we can reduce it by opening the gap as far as the lasing condition is satisfied.

[1] J. Arthur, G. Materlik, A. R. Tatchyn, and H. Winick, Rev. Sci. Instrum. 66, 1987 (1995).

[2] J. Rossbach, Nucl. Instrum. Methods Phys. Res., Sect. A 375, 269 (1996).

[3] T. Shintake, H. Matsumoto, T. Ishikawa, and H. Kitamura, in Proceedings of the SPIE's 46th Annual Meeting, San
Diego, CA, 2001 (SPIE-International Society for Optical Engineering, Bellingham, WA, to be published).

[4] P. Emma, R. Carr, and H. D. Nuhn, Nucl. Instrum. Methods Phys. Res., Sect. A 429, 407 (1999).

[5] U. Hahn, J. Pflüger, and G. Schmidt, Nucl. Instrum. Methods Phys. Res., Sect. A 429, 276 (1999).

[6] E. Jerby and A. Gover, IEEE J. Quantum Electron. 21, 1041 (1985).

[7] K. J. Kim, Nucl. Instrum. Methods Phys. Res., Sect. A 375, 314 (1996).

[8] K. J. Kim, Nucl. Instrum. Methods Phys. Res., Sect. A 407, 126 (1998).

[9] M. Xie, in Proceedings of the Particle Accelerator Conference, Dallas, Texas, 1995 (IEEE, Piscataway, NJ, 1996), p. 183.

[10] L.H. Yu, S. Krinsky, R. L. Gluckstern, and J. B. J. van Zejits, Phys. Rev. A 45, 1163 (1992).

[11] E. L. Saldin, E. A. Schneidmiller, and M. V. Yurkov, Phys. Rep. 260, 187 (1995).

[12] E. L. Saldin, E. A. Schneidmiller, and M. V. Yurkov, Opt. Commun. 148, 383 (1998).

[13] C. Penman and B. W. J. McNeil, Opt. Commun. 90, 82 (1992).

[14] H. Kitamura, T. Bizen, T. Hara, X. Maréchal, T. Seike, and T. Tanaka, Nucl. Instrum. Methods Phys. Res., Sect. A 467-468, 110 (2001). 